\title{
Helping homologues to find their partners
}

DOI:

10.1038/nrg2154

URLs

Entrez

UNC84A

http://www.ncbi.nlm.nih. gov/sites/entrez?Db=gene\&C $\mathrm{md}=$ ShowDetailView\&TermTo Search $=77053$
During meiotic prophase, chromosomes attach to the nuclear envelope through their telomeres, facilitating homologue pairing. Although the first description of this chromosome organization dates back more than a century, the molecular mechanisms are still largely unknown. Two groups now report that, in mice and Caenorhabditis elegans, nuclear inner membrane proteins have an important role in this process.

Min Han and colleagues showed that mice knockout for the nuclear envelope protein SUN1 (also known as UNC84A) develop normally, but both males and females

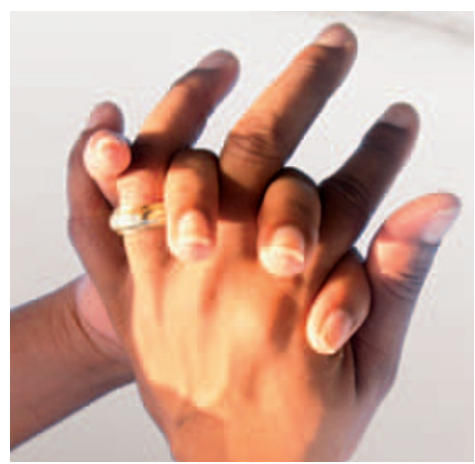

are sterile. SUN1 distribution in wild-type mice varies during the different stages of spermatogenesis, resembling the nuclear envelope localization of telomeres in primary spermatocytes, and fluorescence in situ hybridization (FISH) analysis confirmed that telomeres colocalize with SUN1 during meiotic prophase. Telomere attachment to the nuclear envelope is impaired in Sun $1^{-/-}$mice, resulting in defective pairing, synapsis and recombination between homologous chromosomes.

In a second study, using forward genetic screening in C. elegans, Verena Jantsch and colleagues identified a missense mutation in the matefin gene, $m t f-1 /$ sun-1(jf18), that causes defects in meiotic chromosome segregation. Using FISH, the authors showed that in mutant gonads chromosomes are unable to find their homologous partners, and instead engage in nonhomologous synapsis. Furthermore, these mutants fail to form nuclear envelope aggregates of the ZYG-12 protein, which normally form where proteins of the pairing centre localize. The authors propose a model whereby chromosomes run through a homology check that takes place at SUN-1-ZYG-12 aggregates: if they find their partners, synapsis occurs; otherwise, they keep searching for their homologues. In mtf-1/sun-1(jf18) mutants, the homology check is impaired and nonhomologous synapsis takes place.

Altogether, these studies provide an important addition to our understanding of the processes of chromosome dynamics during meiosis, which might ultimately provide clues about the origins of meiotic defects that impair human fertility.

Francesca Pentimalli

ORIGINAL RESEARCH PAPERS Ding, X. \&

$\mathrm{Xu}$, R. et al. SUN1 is required for telomere attachment to nuclear envelope and gametogenesis in mice. Dev. Cell 12, 863-872 (2007)| Penkner, A. et al. The nuclear envelope protein Matefin/SUN-1 is required for

homologous pairing in $\mathrm{C}$. elegans meiosis. Dev. Cell 12, 873-885 (2007) FURTHER READING Gerton, J. L. \& Hawley, R. S. Homologous chromosome interactions in meiosis: diversity amidst conservation. Nature Rev. Genet. 6, 477-487 (2005) 